\title{
Conociendo la ansiedad matemática. El rol del docente
}

\author{
Knowing math anxiety. The role of the teacher
}

Recibido febrero 2021 Arbitrado marzo 2021 Aceptado abril 2021 Publicado mayo 2021

\author{
Jully Catherine Cordero Arteaga \\ jullycordero@gmail.com \\ Código ORCID: 0000-0003-2487-1575
}

Universidad Pedagógica Experimental Libertador, Caracas, Venezuela

\section{Resumen}

El aprendizaje de las matemáticas no resulta agradable para muchas personas. Se sienten incómodas, desmotivadas, preocupadas e incluso angustiadas ante situaciones que le exigen análisis lógicos y numéricos, influyendo significativamente en el desarrollo educativo del alumno. Esta condición configura la ansiedad matemática. Ante ello, enmarcado en el proceso de enseñanza y aprendizaje de las matemáticas, al docente le corresponde asumir un rol proactivo que favorezca la superación de la dificultad evidenciada, siempre acompañados del estudiante y la familia que constituyen entes insustituibles. Esta reflexión tiene como objetivo brindar la información fundamental para que el profesorado comprenda la ansiedad matemática, reconozca sus causas y consecuencias, adquiera la capacidad para identificarla y adopte y construya diversas estrategias educativas para afrontar con éxito la ansiedad matemática en el aula de clases.

\footnotetext{
Abstract

Learning mathematics is not pleasant for many people. They feel uncomfortable, unmotivated, worried and even distressed in situations that require logical and numerical analysis, significantly influencing the educational development of the student. This condition configures mathematical anxiety. Given this, framed in the process of teaching and learning mathematics, the teacher must assume a proactive role that favors overcoming the difficulty evidenced, always accompanied by the student and the family who constitute irreplaceable entities in the process. This reflection aims to provide fundamental information for teachers to understand math anxiety, recognize its causes and consequences, acquire the ability to identify it, and adopt and build various educational strategies to successfully deal with math anxiety in the classroom.
}

\section{Palabras clave:}

Rol; docente; ansiedad matemática; estrategias educativas 


\section{INTRODUCCIÓN}

$\mathrm{E}$ l aprendizaje de las matemáticas es fundamental en la formación integral del ser humano. En gran parte de las actividades diarias se evidencia el uso de procesos lógicos y numéricos que estructuran el avance en múltiples tareas, tales como comprar, ahorrar, calcular el tiempo para llegar a un lugar, número de personas en un sitio, entender la variabilidad de la temperatura, estimar distancias, etc. El desarrollo del pensamiento lógico constituye la base indispensable para la adquisición de los conocimientos de todas las áreas académicas y es un instrumento a través del cual se asegura la interacción humana (Rodríguez, 2010), es por ello que las matemáticas constituyen una disciplina de orientación prioritaria en los centros educativos. Sin embargo, analizando directamente la práctica en el aula de clases es común notar que no todos los estudiantes logran apropiarse de los conocimientos matemáticos, algunos solo adquieren saberes escasos y limitados.

Las competencias matemáticas que se pretenden desplegar en la escuela han sido establecidas previo al análisis detallado del contexto cotidiano del estudiantado y la revisión y evaluación por parte de expertos, constituyendo una formación básica y necesaria más no implica una exigencia desbordada a la realidad; hasta las instituciones educativas de cada país organizan sus planes de área y diseños curriculares en matemáticas acatando las sugerencias que imparte su gobierno mediante las institucionalidades administradoras de la educación nacional. No obstante, la enseñanza de esta ciencia se ha convertido en un reto para el profesorado, en los colegios es habitual el bajo rendimiento académico de los estudiantes en la asignatura. Aunado a ello, en la comunidad se ha generalizado ese pensamiento que caracteriza a las matemáticas como una disciplina compleja, difícil de comprender y de gran requerimiento mental, producto de creencias cultivadas en el tiempo.

Las calificaciones deficientes en matemáticas pueden ser consecuencia de distintas variables, como el poco afianzamiento de conocimientos previos y fundamentales, hacinamiento, escaso tiempo para la profundización de contenidos, factores económicos familiares, relación del docente y estudiante y demás. Pero, resulta 
preocupante conocer otros comentarios de algunos alumnos con respecto a sus clases de matemáticas, quienes con el simple hecho de recordar que en instantes iniciaría dicha sesión, manifiestan de manera verbal y no verbal el sentimiento de rechazo para con su aprendizaje, representado en preocupación, desmotivación, fobia, frustración y angustia; incluso en algunas situaciones parece que se configurara una condición que afecta significativamente el bienestar físico del individuo, más allá de una percepción desfavorable. Este estado tan peculiar concuerda con la ansiedad matemática, conceptualizada en 1988 por Wood (citado en PérezTyteca, Castro Enrique, Rico y Castro Encarnación, 2011) como la "ausencia de confort que alguien podría experimentar cuando se le exige rendir en matemáticas" (p. 11).

En este contexto, se confirma que en el aula de clases ante el desarrollo habitual de una orientación en matemáticas y de manera involuntaria, se puede presentar afectaciones al estado de ánimo de los educandos sin razón expuesta, configurando la ansiedad matemática. Es imperativo que toda la comunidad educativa sea consciente de la existencia de esta condición y adopte las acciones preventivas y correctivas para evitar y/o mitigar las posibles consecuencias, con el fin de favorecer la formación integral del individuo. Por ello, el propósito de esta investigación es compartir una reflexión de los aspectos relevantes que rodean a la ansiedad matemática, así como definir uno de los papeles protagonistas del proceso educativo ante estas dificultades: el rol docente, tomando como referencia su posición de orientador y facilitador del aprendizaje.

\section{REFLEXIÓN}

¿Qué es la ansiedad matemática?

El ser humano puede reaccionar de múltiples formas frente a escenarios en los cuales no se puede controlar los factores del entorno, algunos actuarán con mayor agilidad, perspicacia, destreza. Otros, serán más cuidadosos, pausados e inclusive podrían atravesar un estado de shock, acompañado de parálisis, sentimientos de angustia, ansiedad, miedo, terror, etc. La sensación de complejidad en estas situaciones es subjetiva, para ciertas personas podría solo presentarse en circunstancias de caso fortuito 
o fuerza mayor. No obstante, otros individuos podrían experimentar ese estado a lo largo de eventos de su vida habitual que simplemente le exigen un mayor esfuerzo: hablar en público, pruebas de evaluación, sustentaciones, entrega de informes a tiempo, cumplimiento de metas y demás. Allí la ansiedad puede parecer inevitable, en el ámbito educativo no es la excepción.

La ansiedad se define como el estado de agitación, inquietud o zozobra del ánimo (Real Academia Española, definición 1). En la escuela, esta se puede experimentar al desarrollar actividades que demanden el empleo de habilidades específicas cómo en los exámenes, competencias, torneos, exposiciones orales, participación al tablero, debates, etc. La ansiedad es un factor afectivo presente en los estudiantes, sobre todo en ambientes de evaluación o al enfrentarse a asignaturas especialmente difíciles para ellos, como pueden ser las matemáticas (Pérez-Tyteca et al., 2011).

El concepto de ansiedad matemática no está unificado, sin embargo a lo largo de los años los investigadores han coincidido en ciertos aspectos. Para Fennema y Sherman (1976) la ansiedad matemática consiste en una serie de sentimientos de ansiedad, terror, nerviosismo y síntomas físicos relacionados que surgen al trabajar con matemáticas. Richardson y Suinn (1972) consideran que es un sentimiento de tensión y ansiedad que interfiere en la manipulación de números y en la resolución de problemas matemáticos en una amplia variedad de situaciones tanto cotidianas como académicas. Tobias y Weissbrod (1980), mencionan que la ansiedad matemática describe el pánico, indefensión, parálisis y desorganización mental que surgen cuando a un sujeto se le exige resolver un problema matemático. Pérez-Tyteca, Monje y Castro (2013), la definen como un estado afectivo caracterizado por la ausencia de confort que puede experimentar un individuo en situaciones relacionadas con las matemáticas, tanto de su vida cotidiana como académica.

Una afirmación indiscutible es que la ansiedad matemática produce en la persona que lo sobrelleva, la intención de evitar a toda costa caer en ambientes que le involucren acudir al razonamiento matemático, anhelando huir de esos espacios y prefiriendo centrar su interés en asuntos de estudio totalmente ajenos a ello. 
Para afrontar efectivamente la ansiedad matemática surge la necesidad de definir las causas de su existencia y así evitar esos agentes detonantes.

\section{¿Cuáles son las causas de la ansiedad matemática?}

Baroody (1988) menciona que el origen de la ansiedad matemática radica en el sistema de creencias del alumno, tendiente a exagerar las demandas insustituibles para hacer frente a las tareas y subestimar sus habilidades de tal manera que esa percepción de incapacidad para solucionar problemas desencadena múltiples emociones negativas, angustiosas y amenazantes.

Por otro lado García (2016), propone una clasificación de dos grandes tipos de elementos intervinientes: los factores ambientales, asociados a las principales variables presentes en el entorno del alumno como son la familia, los profesores y el sistema educativo, cuyas actitudes, estereotipos y estilos de enseñanza influyen significativamente en las expectativas y reacciones del estudiante; y los factores personales, en los cuales incluye variables emocionales vinculadas a los afectos y gustos propios del estudiante y variables cognitivas relacionadas con habilidades de pensamiento abstracto, la cognición numérica o capacidad de la memoria de trabajo.

Al respecto Villamizar, Araujo y Trujillo (2020), expresan que:

La ansiedad matemática es una situación especial que se presenta cuando se conjugan factores de personalidad, ambientales e intelectuales. Entre los factores de personalidad se encuentran la baja autoestima y el temor para preguntar; entre los ambientales, las experiencias negativas en el aprendizaje de las matemáticas, y las actitudes negativas tanto de padres de familia como de profesores; y entre los intelectuales, la sensación de incompetencia para aprender matemáticas, la falta de utilidad percibida de las matemáticas y la no coordinación entre los estilos de enseñanza de los profesores y los estilos de aprendizaje de los estudiantes. (p. 2-3)

En este orden de ideas, es preciso destacar que existen opiniones variadas con respecto a las causas de la ansiedad matemática, pese a ello en los últimos años se concuerda en la atribución de un origen multifactorial. También, es indudable que al involucrar el ámbito emocional y afectivo del individuo (lo que 
constituye la personalidad, identidad e intereses del alumno, los cuales inciden de manera relevante en el posible desarrollo de la ansiedad matemática) se intensifica la complejidad del proceso cuyo objetivo sea disminuir la ansiedad matemática, ya que conlleva lograr influenciar al individuo en pro de modificar su voluntad y esto solo se obtiene luego de que el estudiante sea consciente de la condición que posee y realmente desee mejorar por sí mismo.

\section{¿Cuáles son las consecuencias de la ansiedad matemática?}

Muñoz y Mato (2007), manifiestan que muchos investigadores han escrito sobre ello, coincidiendo en los siguientes efectos: la incapacidad para resolver operaciones matemáticas, disminución del rendimiento académico en matemáticas, evitar matricularse en cursos, itinerarios o carreras universitarias que relacionen estos saberes y los sentimientos negativos de culpa y vergüenza.

Luego de revisar múltiples estudios previos, Delgado, Espinoza y Fonseca (2017), agregan que la ansiedad matemática provoca en los alumnos la adopción de determinadas conductas cuando se trabaja en contenidos matemáticos, tales como la disminución de energía y atención en actividades cognitivas y la realización de las operaciones sin cuidado, con el objetivo de terminar pronto la tarea asignada para reducir el tiempo de incomodidad al que se sienten expuestos.

Sagasti (2019), puntualiza que la ansiedad matemática tiene consecuencias negativas para el desarrollo de las matemáticas, la educación matemática y la participación de adultos en actividades relacionadas con las matemáticas.

Es un panorama lamentable el que deriva de la ansiedad matemática, considerando especialmente la transversalidad de las matemáticas en los diferentes campos científicos. Los estudiantes con esta dificultad no se apropian de los saberes matemáticos fundamentales para enfrentar retos venideros en su contexto personal, académico y profesional, incluso pretenden abandonar totalmente el aprendizaje de este pilar esencial del conocimiento humano.

\section{Entonces, ¿Las matemáticas son complejas?}

Por su naturaleza de exactitud y objetividad, las matemáticas requieren dedicación y compromiso, más esto no debe representar 
obstáculo alguno. Muchas personas disfrutan y se apasionan con el aprendizaje y enseñanza de las matemáticas. Hidalgo, Maroto y Palacios (2005) mencionan que "las matemáticas son una disciplina que requiere para su asimilación cierto esfuerzo y el uso de estrategias cognitivas de orden superior. A ello, se suma el hecho de que los aprendizajes matemáticos son acumulativos, como lo son también las dificultades" (p. 90). Esto confirma que los estudiantes perciben un reto ante el aprendizaje de las matemáticas, desafío que comparten los docentes de matemáticas desde la enseñanza de las mismas. Para obtener un resultado satisfactorio en este proceso, se exige la eliminación de esas barreras que se han plantado en lo más profundo de la mente de los alumnos: la ansiedad matemática.

¿Cuál es el rol del docente frente a la ansiedad matemática?

El docente y el sistema educativo en el cual este se apoya, también ocupan un lugar sobresaliente como factores influyentes en la generación de la ansiedad matemática, es ese el espacio que se debe explotar para promover sensaciones inversas, es decir favorecer el amor e interés para con las matemáticas y prevenir, disminuir o eliminar los niveles de ansiedad matemática en el estudiantado. De allí que conviene promover un rol activo y protagónico del personal docente, inicialmente identificando a los alumnos que viven con esta condición.

¿Cómo identificar a los alumnos que viven con ansiedad matemática?

El diagnóstico de esta dificultad por parte del profesor en el aula de clases, se produce desde la observación participativa y el seguimiento del desempeño integral del alumno, además de sus reportes de calificaciones académicas se debe tener en cuenta sus actitudes hacia el aprendizaje de las matemáticas. Siendo realistas, esta actividad puede resultar compleja, especialmente en las escuelas públicas, debido al tiempo que representa este análisis y la necesidad de atención a los detalles, en coherencia con la enorme cantidad de estudiantes y las horas estrictas de clase para cada grupo de alumnos.

Existen instrumentos de evaluación como herramientas de apoyo para detectar esta condición, los cuales favorecen la practicidad, agilidad y aportan objetividad al resultado. Los 
instrumentos que se han establecido en el campo científico para evaluar la ansiedad matemática son variados y originalmente se encuentran en idioma inglés, a pesar de que se han adaptados algunos al castellano, ciertos apartes podrían confundir al sujeto evaluado esencialmente porque hay frases en sentido negativo.

Para medir el nivel de ansiedad matemática en los alumnos conforme al idioma español, se resalta la adaptación al Castellano de la Escala de Ansiedad Matemática de Fennema-Sherman (1976). La escala se ha validado desde el momento de su creación y a lo largo de sus 30 años de aplicación. Su fiabilidad se comprobó en el momento de su construcción; el índice de fiabilidad obtenido es 0,75, que se considera aceptable (Pérez-Tyteca et al., 2011).

Este instrumento es un cuestionario tipo Likert, formado por un total de 12 ítems:

1. No le tengo ningún miedo a las matemáticas

2. No me importaría hacer más cursos de matemáticas

3. Normalmente no me preocupo con relación a si soy capaz de resolver los problemas de matemáticas

4. Casi nunca me pongo nervioso durante un examen de matemáticas

5. Normalmente estoy tranquilo durante los exámenes de matemáticas

6. Normalmente estoy tranquilo en las clases de matemáticas

7. Normalmente las matemáticas me ponen incómodo y nervioso

8. Las matemáticas me ponen incómodo, inquieto, irritable e impaciente

9. Me pongo mal cuando pienso en intentar hacer problemas de matemáticas

10. Cuando hago problemas de matemáticas se me queda la mente en blanco y no soy capaz de pensar claramente

11. Una prueba de matemáticas me daría miedo

12.Las matemáticas me hacen sentir preocupado, confundido y nervioso

Cada ítem cuenta con 5 posibilidades de respuesta: desde "totalmente en desacuerdo" a "totalmente de acuerdo", incluyendo una respuesta neutra correspondiente a "ni de acuerdo ni en desacuerdo". La codificación de las 5 posibles respuestas va del 1 al 
5, por lo que la puntuación media obtenida puede variar desde 1 a 5 puntos, previa recodificación de los ítems negativos en escala inversa.

Para interpretar los resultados Pérez-Tyteca (2012) establece las siguientes categorías:

- Ansiedad muy baja: Puntuación media menor a 1,5.

- Ansiedad baja: Puntuación media entre 1,5 y 2,49.

- Ansiedad media: Puntuación media entre 2,5 y 3,49.

- Ansiedad alta: Puntuación media entre 3,5 y 4,49.

- Ansiedad muy alta: Puntuación media mayor o igual a 4,5.

- Luego del análisis e interpretación de los datos, se podrá identificar a los alumnos que poseen un nivel de ansiedad matemática elevado.

¿Qué procedimiento debe adoptar el docente ante estudiantes con ansiedad matemática?

Prosigue el reconocimiento de las causas particulares que podrían haber provocado la ansiedad matemática, labor que se espera sea abanderada por el profesor, no obstante amerita la cooperación del mismo estudiante y la intervención de la familia, para así precisar de manera efectiva la trazabilidad de la situación, facilitando el diseño e implementación de procedimientos que realmente atiendan la circunstancia específica. Una de las técnicas de recolección de información más efectivas en estos casos son las entrevistas con el alumno, familiares, compañeros y docentes.

Según los datos obtenidos, el docente como ente facilitador del aprendizaje podrá generar estrategias de apoyo, cuya meta será disminuir los niveles de ansiedad matemática y estas serán incorporadas en un programa de actividades. Las actividades se plantearán con tendencias a fortalecer la autoestima del alumno, fomentar la expresión de sus sentimientos, incentivar el optimismo, incrementar su confianza, fortalecer los hábitos de estudio, autorreflexión, etc.

Para el diseño de cada una de las actividades se debe tener en cuenta la delimitación de los siguientes aspectos: objetivo específico, materiales, lugar, duración y descripción; todo ello enmarcado en un cronograma, al cual se le debe efectuar el seguimiento periódico respectivo verificando el cumplimiento de lo 
proyectado. Es muy importante que luego de aplicado el programa de actividades, se ejecute nuevamente la medición del nivel de ansiedad matemática al alumno, para corroborar la efectividad de las tareas implementadas y si se hace necesario elaborar un nuevo programa.

¿Y si se evidencia un nivel de ansiedad matemática con dificultad alta?

Cabe precisar que si a lo largo del proceso se logra notar un nivel de complejidad desconcertante, el caso debe ser remitido a la orientación escolar porque ya sobrepasa el alcance formativo del educador y para ello existen procedimientos especiales a cargo de los expertos en el área.

¿Qué estrategias generales son útiles para prevenir, disminuir o eliminar la ansiedad matemática?

Como proceso habitual para cada profesor, se les insta a que consideren que conforme a las características de su grupo de estudiantes, entorno escolar, sus propias destrezas, concepciones pedagógicas, experiencia y estructura curricular, establezcan su metodología de enseñanza. Adicionalmente, tomen en cuenta evitar el desarrollo de ese enemigo distractor que representa la ansiedad matemática y se mantengan en continúa actualización de la práctica formativa. Las actuales perspectivas educativas integradoras del proceso de enseñanza y aprendizaje, procuran abordar el ámbito cognitivo y afectivo del estudiante, partiendo de su interacción con el contexto social, cultural y escolar. (Caballero, Cárdenas y Gordillo, 2016).

Woodard (2004), invita al profesorado a mantener actualizado sus conocimientos con respecto a la ansiedad matemática, leyendo literatura relacionada y participando en espacios académicos sobre ello. También añade orientaciones para los profesores que incluyen: ser más flexibles en la evaluación usando diversas técnicas (preguntas orales, observación, demostración, discusión, escritura en un diario, reevaluación, proyectos) verificando en los exámenes los procedimientos además de la respuesta y ofreciendo tiempo de prueba alternativos, implementar el uso de calculadoras gráficas y otros instrumentos tecnológicos, asegurar que sus explicaciones sean de manera clara y sencilla, revisar los conceptos básicos, 
incentivar la enseñanza del pensamiento crítico, demostrar entusiasmo por el tema y dar retroalimentación.

Barnes (citado por Caballero, Cárdenas y Gordillo, 2016), recomienda que los profesores han de enseñar hábitos de estudio, acrecentar la autoconfianza en las habilidades matemáticas, moverse a lo largo del aula para apoyar a los estudiantes y responder sus preguntas y plantear más actividades prácticas ajustadas al contexto real. Igualmente, resalta que los alumnos valoran la tutoría en otros espacios luego de la clase y la relajación.

Edelmuth (citado por Monje, Pérez y Castro, 2012), expone una serie de técnicas dirigidas a los alumnos, a los padres y a los profesores.

Para los docentes aconseja crear un ambiente distendido en clase en el que los alumnos se sientan confiados y tratar cuestiones de una manera abierta, haciendo uso del humor y mostrando a los estudiantes que existen diferentes caminos para obtener la solución de un problema y que los errores dan la oportunidad de aprender. Por otra parte, aconseja a los padres educar a sus hijos en la cultura matemática propiciada al tratar con ellos, siempre que sea posible, problemas matemáticos presentes en la vida diaria (contar, comparar precios, razonar de manera lógica ante ciertas situaciones, etc.). (p. 51-52)

Iossi (2007), realiza una revisión de la literatura con el fin de explorar estrategias para minimizar la ansiedad matemática, clasificándolas en tres grupos: (a) estrategias curriculares, como reevaluación o repetición de exámenes, aprendizaje autónomo al ritmo del estudiante, educación a distancia, clases con estudiantes del mismo sexo y cursos para minimizar la ansiedad matemática, (b) estrategias instruccionales, aquellas que son incorporadas o motivadas por el docente tales como los materiales prácticos y manipulativos, integración de la tecnología en la enseñanza de las matemáticas, técnicas de autorregulación para mantener una actitud positiva y comunicación flexible y fluida, (c) estrategias no instruccionales, las cuales se encuentran fuera del salón de clases y no involucran la experiencia docente, como las técnicas de relajación (meditación, yoga y psicoterapia) y el tratamiento psicológico. 
Beilock y Willingham (2014), responden la pregunta ¿Qué pueden realizar los maestros con relación a la ansiedad matemática?, destacando que los docentes pueden asegurar el aprendizaje de las habilidades matemáticas fundamentales fomentando la participación de los padres, identificar a los estudiantes en riesgo de desarrollar ansiedad matemática, asignar actividades para el perfeccionamiento de las competencias matemáticas básicas, adoptar diferentes metodologías de evaluación, promover ejercicios de escritura libre donde el alumno exprese sus emociones, mantener una comunicación asertiva especialmente cuando los estudiantes manifiestan dificultades. Igualmente suscita el fortalecimiento del entrenamiento en los profesores para afianzar su confianza al enseñar matemáticas.

Las estrategias de enseñanza que definitivamente integre el docente para prevenir, mitigar o eliminar la ansiedad matemática dependerán del entorno en el que se encuentre, las ideas previamente socializadas pueden servir de referente más no constituyen una lista cerrada de opciones. Por supuesto, de estas estrategias de enseñanza sugeridas se destacan tres aspectos que merecen atención al momento de formalizar la metodología de enseñanza: (a) estabilidad emocional y actitud hacia el aprendizaje del estudiante, que implica fortalecer la seguridad en sí mismo, incentivar la consolidación de hábitos de estudio y promover relaciones interpersonales sanas tanto con el docente como con sus compañeros, (b) reforzar los conocimientos y habilidades básicas matemáticas, aunque parezcan ser demasiado sencillos o que ya han sido previamente interiorizados por el alumno, (c) para la enseñanza de las nuevas competencias matemáticas es importante demostrar la concatenación con los saberes previos, implementar el uso de herramientas tecnológicas, evidenciar la aplicabilidad en el contexto cotidiano, apoyarse en la lúdica y diversificar las técnicas de evaluación.

Igualmente, resultaría muy fructífero que los docentes en cada institución educativa conformen equipos de trabajo, de los cuales resulten estrategias grupales, directrices procedimentales, experiencias significativas que favorezcan la afrontación y eliminación de la ansiedad matemática. La retroalimentación efectiva de las prácticas compartidas es enriquecedora y 
provechosa, abarca perspectivas ideológicas variadas, con mayor cobertura e impacto.

¿Cuál es el rol de la familia ante la ansiedad matemática?

A pesar de que esta reflexión se centró en el rol del docente, se ha podido visibilizar el papel irremplazable de la familia. Es conocido que esta constituye la institución básica de la sociedad, representa el avistamiento inicial y el contacto primario de las personas con el mundo, posteriormente es que se configuran las relaciones con amigos y la escuela. De allí, que les concierne fomentar la autonomía de los niños, su equilibrio emocional y la capacidad de establecer vínculos personales satisfactorios. La familia posee una función como ente socializador de los valores colectivamente aceptados y la enculturación.

Frente el ámbito escolar, el núcleo familiar debe participar enérgicamente en las actuaciones que se emprendan en las instituciones escolares a través de los docentes, mantener una disposición de colaboración y acompañamiento, proporcionar las condiciones y recursos necesarios, motivar a que los niños estudien y adquieran destrezas e incentivar el respeto para con la comunidad educativa y sociedad en general.

\section{CONCLUSIONES}

L a ansiedad matemática constituye una realidad que se debe afrontar, es un concepto que debe ser conocido y asumido con el mayor compromiso por parte de la comunidad educativa. El rol del docente es fundamental para enfrentar esta condición, son entes buscadores y generadores de conocimiento, se caracterizan por su pasión para servir a la sociedad y el deseo de orientar al máximo a sus alumnos, no solo en el contexto disciplinar sino en todas las esferas de su vida. Por ello, se hace necesario que el profesorado conozca los aspectos fundamentales que envuelve la ansiedad matemática y sea consciente de su extensión, reconociendo su manifestación en los alumnos a través de sentimientos de angustia, preocupación, miedo ante la solicitud de aplicar las matemáticas; comprendiendo su origen multifactorial que tiene como consecuencia el bajo desempeño en matemáticas. Para confirmar la presencia de la ansiedad matemática en el 
estudiante, se recurre a la aplicación de instrumentos de evaluación que generan resultados objetivos. Así se podrá construir un programa de actividades que favorezcan la superación de esta dificultad. También, los docentes pueden adoptar diversas estrategias generales en su metodología de enseñanza que minimicen el riesgo de poseer ansiedad matemática.

Cabe destacar, que el apoyo de los maestros ha favorecido en la evolución del ser humano y la ansiedad matemática es solo un impasse que puede ser superado con su acompañamiento, siempre y cuando el estudiante y su familia también se involucren fielmente en el proceso de enseñanza y aprendizaje de las matemáticas. Es preciso recordar que finalmente en el proceso de aprendizaje, el estudiante es el protagonista principal, que lleva consigo un aspecto determinante en todo este contexto: la voluntad de aprender. No tiene utilidad alguna aplicar múltiples y novedosas estrategias de enseñanza, si el alumno no está en disposición de adquirir un saber.

\section{REFERENCIAS}

Baroody, A. (1988). El pensamiento matemático de los niños: Un marco evolutivo para maestros de preescolar, ciclo inicial y educación especial. Madrid: Visor, Centro de Publicaciones del M.E.C

Beilock, S. y Willingham, D. (2014). Ask the cognitive scientist-math anxiety: can teachers help students reduce it. American Educator, 38 , 28-33. https://files.eric.ed.gov/fulltext/EJ1043398.pdf

Caballero, A., Cárdenas, J. y Gordillo, F. (2016). La intervención en variables afectivas hacia las matemáticas y la resolución de problemas matemáticos. El MIRPM. En Berciano, Ainhoa; Fernández, Catalina; Fernández, Teresa; González, José Luis; Hernández, Pedro; Jiménez, Antonio; Macías, Juan Antonio; Ruiz, Francisco José; Sánchez, María Teresa (Eds.), Investigación en Educación Matemática XX, 75-91. Universidad de Málaga, España. https://www.seiem.es/docs/actas/20/ActasXXSEIEM.pdf

Delgado, I., Espinoza, J. y Fonseca, J. (2017). Ansiedad matemática en estudiantes universitarios de Costa Rica y su relación con el rendimiento académico y variables sociodemográficas. Propósitos y Representaciones, 5(1), 275-324. http://revistas.usil.edu.pe/index.php/pyr/article/view/148/249 
Fennema, E. y Sherman, J. (1976). Fennema-Sherman Mathematics Attitudes Scales: Instruments Designed to Measure Attitudes toward the Learning of Mathematics by Females and Males. Journal for Research in Mathematics Education, 7(5), 324 - 326

García, A. (2016). Ansiedad a las matemáticas. [Trabajo de grado no publicado, Universidad de Jaén] Repositorio facultad de humanidades y ciencias de la educación. http://tauja.ujaen.es/bitstream/10953.1/4092/1/Garca_Lpez_Aroa TFG_Grado Primaria.pdf

Hidālgo, S., Māroto, A. y Palacios, A. (2005). El perfil emocional matemático predictor de rechazo escolar: Relación con las destrezas y los conocimientos desde una perspectiva evolutiva. Educación matemática, 17(2), 89-11. https://www.redalyc.org/articulo.oa?id=40517205

Iossi, L. (2007). Strategies for reducing math anxiety in postsecondary students. In S. M. Nielsen \& M. S. Plakhotnik (Eds.), Proceedings of the Sixth Annual College of Education Research Conference: Urban and International Education Section. Miami, Florida International University. https://digitalcommons.fiu.edu /cgi/viewcontent.cgi?referer=https://www.google.com/\&httpsred ir $=1$ \&article $=1257 \&$ context $=$ sferc

Monje, J., Pérez, P. y Castro, E. (2012). Resolución de problemas y ansiedad matemática: profundizando en su relación. Revista Iberoamericana de Educación Matemática 32(1), 45-62. http://www.fisem.org/www/union/revistas/2012/32/archivo7_volu men32.pdf

Muñoz, J. y Mato, M. (2007). Elaboración y estructura factorial de un cuestionario para medir la "ansiedad hacia las Matemáticas" en alumnos de Educación Secundaria Obligatoria. Revista galego-portuguesa de Psicoloxía e Educación: revista de estudios e investigación en psicología y educación, 14, 221-231. http://ruc.udc.es/dspace/handle/2183/7064

Pérez-Tyteca, P., Castro, E., Rico, L. y Castro, E. (2011). Ansiedad matemática, género y ramas de conocimiento en alumnos universitarios. Enseñanza de las ciencias, 29(2), 237 - 249. https://www.raco.cat/index.php/Ensenanza/article/view/243835/3 53438

Pérez-Tyteca, P. (2012). La ansiedad matemática como centro de un modelo causal predictivo de la elección de carreras. (Tesis Doctoral). Universidad de Granada, España. https://hera.ugr.es/tesisugr/2108144x.pdf

Pérez-Tyteca, P., Monje, J. y Castro, E. (2013). Afecto y matemáticas. Diseño de una entrevista para acceder a los sentimientos de alumnos adolescentes. AIEM. Avances de 
Investigación en Educación Matemática, 4(1), 65 - 82. https://documat.unirioja.es/descarga/articulo/5421236.pdf

Real Academia Española (s.f). Diccionario de la lengua española, 23 a ed., [versión 23.4 en línea]. https://dle.rae.es. Recuperado el 07 de Mayo de 2021.

Richardson, F. y Suinn, R. (1972). The Mathematics Anxiety Rating Scale: Psychometric data. Journal of Counseling Psychology, 9(6), 551 - 554.

Rodríguez, M. (2010). La matemática: ciencia clave en el desarrollo integral de los estudiantes de educación inicial. Zona Próxima, 13(1), $130 \quad 141$ http://www.redalyc.org/articulo.oa?id=85317326009

Sagasti, M. (2019). La ansiedad matemática. Matemáticas, $\begin{array}{llll}\text { Educación } \quad y \quad \text { Sociedad, } & \text { 2(2), }\end{array}$ https://www.researchgate.net/publication/335842850_La_ansied ad matematica

Tobiass, S. y Weissbrod, C. (1980). Anxiety and Mathematics: An Update. Harvard Educational Review, 50(1), 63 - 70.

Villamizar, G., Araujo, T. y Trujillo, W. (2020). Relación entre ansiedad matemática y rendimiento académico en matemáticas en estudiantes de secundaria. Ciencias Psicológicas, 14(1), e2174. http://www.scielo.edu.uy/pdf/cp/v14n1/1688-4221-cp-1401-e2174.pdf

Woodard, T. (2004). The Effects of Math Anxiety on Post-Secondary Developmental Students as Related to Achievement, Gender, and Age. Inquiry: Critical Thinking Across the Disciplines, 9(1). https://files.eric.ed.gov/fulltext/EJ876845.pdf 